\title{
Developing and Implementing Machine Learning Software at Home Depot
}

\author{
Herbert Remidez, University of Dallas, USA*
}

Sri Beldona, University of Dallas, USA

\begin{abstract}
This teaching case explores the problem of shelfouts and the use of technology adoption to minimize it. Shelfout, wherein a product is not on the shelf when it is supposed to be, has received renewed interest especially given the fact that many brick-and-mortar stores shut down due to their inability to compete with online vendors. The coronavirus pandemic worsened this problem and companies continue to struggle with the resulting supply chain disruptions. Increasingly consumers are searching for products on the website to confirm product availability before traveling to the store. In this case, the authors show how The Home Depot, Inc. (Home Depot) is addressing shelfouts and the process they went through in solving this problem. Instructors can use this case to introduce students to the machine learning development lifecycle, marketing courses discussing shelfouts, and courses with lessons related to technology implementation.
\end{abstract}

\section{KEYWORDS}

Cloud Computing, Data Pipeline, Hybrid Cloud, Machine Learning, Supply Chain Management, System Implementation

\section{INTRODUCTION}

The Home Depot, Inc. (Home Depot), was founded in 1978 and is the number one home improvement retailer in the world. The first stores opened in Atlanta in 1979 with 60,000 square feet and stocked about 25,000 products. The company has since grown to more than 2,200 stores across the United States, Canada, and Mexico, and it employs more than 400,000 associates and has over $\$ 100$ billion in annual revenues. The average store has 100,000 square feet of indoor retail space and has 33,000 unique products or stock-keeping units (SKUs). Its eCommerce offering has more than one million products. The company's vision is to become a one-stop-shop for do-it-yourselfers, contractors, and installation businesses.

The founders of Home Depot created a customer "bill of rights" that stated, “...customers should always expect the best assortment, quantity, and price, as well as the help of a trained associate when they visit a Home Depot store" (Home Depot, 2019). The Home Depot's strategic framework is based on the tenets of customer experience, product authority, and capital allocation driven by productivity and efficiency and interconnected retail. A guiding strategic principle of such customer service starts with the product being in stock and on the shelf. 


\section{BACKGROUND}

This section provides an overview of the problem, the origins of the project, and how the team tested a proposed problem solution before initiating the full project.

\section{The Shelfout Problem}

Home Depot customers can view products on the company website to check if their local store has the product(s) they need before traveling to the store to make a purchase. The website provides an estimate of the number of items of the products available and in which aisle and bay the items are located based on shipment and sales data. These are just estimates and can cause customers to arrive at the store only to find products unavailable. The website estimate is correct in estimating that the product is in the store but might not be on the shelf where the customer expects to find it. The product is in the store but not on the right shelf (Chuang, 2018). This is a "shelfout" problem or shelf outof-stock problem, which is distinct from an "out of stock" problem, where the product is not in the store. Customers in this situation can either choose a similar product that is available or leave the store without making a purchase. The problem of a store's inventory system showing a product is in stock when it is not on the shelf in the correct location affects all customers, regardless of whether they checked online beforehand or not.

Customers can also contribute to the shelfout problem and are associated with two of the three major causes of shelfouts. When searching for a product, customers sometimes place another product in front of a product, which hides the product from customers or associates seeking the product. Customers selecting a product, carrying it with them to another part of the store, deciding not to buy the product, and leaving the product at another point in the store is another cause of shelfouts. The third major reason for shelfouts is when the number of items shipped to a store exceeds the shelf space allocated to the item. This leads to the shelf being fully stocked and the remaining items being placed in an overhead bin, where they might not be easily found but showing as in the store by the inventory system.

The shelfout problem is difficult to solve because each Home Depot store is a working warehouse, which means that all the inventory is on the floor, and there is not much backroom storage. Also, the store environment is dynamic, and many teams share responsibility for ensuring product availability. The Freight Team (FT) oversees stocking the shelves from the receiving trucks, which usually arrive at night. FT members place any products that cannot fit on the shelf on an overhead shelf. Merchandise Execution Teams (METs) work during the day and oversee pack downs, which is the process of moving products from the overhead space to the proper shelves and pulling items to the front of a shelf so that is easy to see and reach (Charrington, 2018; Google, 2018). Pack downs occur at specific intervals throughout the day. Associate team members work on the store floor helping customers and assisting with keeping the store neat. Customers know that a Home Depot store is a working warehouse and often assume a product is not available if it is not on the shelf.

This case describes a project addressing the shelfout problem at The Home Depot. The team responsible for the project chose to implement a limited set of features for the first version of the system. With the first phase completed, the team embarks on a journey of reflection on the lessons learned and options for expanding the project. The team also identifies important considerations for managers preparing to undertake big data analytics projects. Specifically, what lessons should the team carry into future projects? What are some of the unique aspects of this project that led to the project's success?

\section{Project Origins}

The Applied Machine Intelligence (AMI) group was formed about a year before the start of this project. The short tenure left them looking for a "quick win" to build credibility and show their value. The team functions as an internal consultant that can build a proof of concept (POC) projects compared 
to a group dedicated to taking projects from inception through production implementation, although they have the capability to build production-ready systems. The project originated when the AMI group was discussing ideas for projects. A data scientist on the team with a background in supply chain management proposed using signals from within the store to predict shelfouts.

\section{Hypothesis Testing}

Not all problems are worth solving. Before the AMI group attempted to solve the shelfout problem, they needed to collect data to help them determine if reducing shelfout was worth the effort. The premise was that increased shelf availability/reducing shelfout translates into an increase in sales. To test this premise, the AMI group designed an experimental test in a few stores using a control group and a treatment group.

For the treatment group of stores, associates went around the store three times a day and pulled all misplaced items from around the store and from the overhead bins and put the items back on the shelves to make sure that shelves were fully stocked and inventory in the store was on the appropriate shelf and easily available to customers. This allowed the AMI team to measure the relationship between increased shelf availability and sales. Because of the proprietary nature of the data, Home Depot did not disclose the result of this experiment. However, they disclosed that increasing shelf availability by one percent was enough to produce a "meaningful increase" in sales. The results provided the required evidence to convince management to support continuing the project, which led to the first pilot test.

\section{PROJECT INITIATION}

Having defined the problem (shelfouts) and conducted a labor-intensive experiment, the team proceeded to initiate the development of a new software system over the period of May 2017 through July 2018. This section describes the development process for data collection to system implementation.

\section{Data Collection and First Pilot Test (May through September)}

Data collection started with a pilot test called "on shelf availability" (OSA). This was a manual approach to understanding the shelfouts by sampling a set of representative stores. The first step was to have the Merchandise Execution Teams (MET), which manage pack downs, scan any empty space they saw on the shelf using a scanner app. The MET did this for each bay for two weeks. The data collected by the MET allowed the AMI group to know which items are typically experiencing shelfout in the store, a piece of vital information the company did not previously have. Although this was manual data collection, it did not add much overhead cost, because MET members are already at these aisles spending anywhere from 10 to 15 minutes doing the pack downs. The addition of the scanning process only took about a minute.

After having the MET collect data and securing data from other systems (e.g., point of sales, supply chain management, space planning, etc.), the AMI group built its first pilot model. The AMI group created the models on their local laptops, generated the output, and asked store managers to physically check the identified items to validate the predictions. AMI set up weekly meetings with the store managers and created surveys to collect the manual validation data and evaluate the quality of their models throughout the development process.

The first model implemented was a Random Forest model developed using the scikit-learn Python library. This manual process showed that the model was working well in predicting shelfouts. The team switched to an XGBoost model during this pilot phase and continued to improve its predictions. This phase was a precursor to setting up data pipelines and building the entire process using the Google Cloud platform. While building the data pipelines to systematically collect and process data from systems scattered throughout the company, the team continued to experiment with incorporating new features into the model. 


\section{Feature Engineering and Model Selection (October through January)}

As the validation phase for the first model concluded, the team turned its attention to feature engineering. Feature engineering is the process of preparing the data to increase performance of the selected algorithm (in this case, XGBoost). The AMI group started by using the data from their existing Enterprise Data Warehouse (EDW) along with the shelfout status data collected from the MET. An EDW is a database holding historical company records from different systems (e.g., human resources, finance, supply chain, sales, etc.). EDWs are critical to the success of big data projects in many industries (Brunello et al., 2019). Next, the team asked for help from key stakeholders across the company to identify potentially valuable features to include in the model. The AMI group collaborated with teams from many departments to collect suggestions for features and the data needed to represent those features. Novices to machine learning might assume developers feed an algorithm all available data and out comes a usable model, but that is far from the truth. This project illustrates how obtaining support from many stakeholders is important to the success of projects like this one. The final model consisted of more than 50 features from a diverse set of systems. Two of the key features included in the final model are the number of items sold at the store compared to the number of items forecasted to sell at the store within a given timeframe and the number of items contained in the package shipped to a store compared to the number of items a shelf can accommodate.

\section{Sales and Forecast}

Data that combined recent sales of a product with sales forecasts were some of the strongest predictors of shelfouts. If the forecast system estimates that an item will have a high rate of sales, and the inventory system indicates the item should be available but the point-of-sale system does not indicate any sales occurring, this might be an indication the item is not available on the appropriate shelf. Sales forecast and sales are dynamic and store specific. This is an example of how combining data from multiple systems to create a single feature (e.g., UnexpectedSalesVolume) for the model can be valuable.

\section{Ratio of Pack Size to Shelf Capacity}

Another example of a key feature was the relationship between the number of items that arrive in a package (i.e., pack size) and the shelf capacity. The combination of these two variables proved to be an informative feature. Pack size can vary between stores and over time. Suppliers can change how many items they include in a package, and not all stores receive supplies from the same supplier. Like pack size, shelf capacity is dynamic. The group responsible for store layout is constantly evaluating how much space to allocate to a product and where to place the product. Sometimes retailers receive a pack of items that contains more items than the shelf can accommodate. For example, the night before, the store receives eight units of an item, but the shelf capacity for that item is only six units. A FT associate will put six units on the shelf and place the remaining two items in the box in an overhead shelf. Sometimes these boxes will have no labels and cannot fit on the overhead shelf near the original item. In such instances, only the associate who stored the box with the two items knows the existence and location of the box containing the extra items. When the store sells through six units on the shelf, in this example, the product is at the risk of experiencing a shelfout. In this case, the ratio of pack size to shelf capacity is a feature that takes data from different systems. Combining the following data points: the size of the actual item (small items are easier to misplace), how much shelf capacity was allocated for the item, the last time the item was received from the vendor, number of items sold since the time the item was received, and the pack size and the shelf capacity creates features the model relied on to estimate the probability of an item experiencing a shelfout incident.

\section{Precision-Recall Tradeoff}

The AMI group confronted the precision-recall dilemma when implementing the model. The AMI group used a binary classification model with a "Yes" or "No" variable to record if a product was 
experiencing a shelfout. The model assigned a score for each item. The score assigned to an item can vary from zero to one, with a higher score indicating an increased likelihood the item was experiencing a shelfout incident. Typically, when using binary classification models, cases with a score at or above 0.50 are labeled as positive instances. In this case, the default would be to ask a MET member at a store to check an item if the model assigned the item a score of 0.50 or greater. If the AMI group asked the MET members to check every item the model assigned a score of 0.50 or above, they ran the risk of having its predictions ignored because an item with a score of 0.51 might not be experiencing a shelfout. Alternatively, if the AMI group was overly cautious and only asked the MET to physically check items the model scored as 0.90 or above, they ran the risk of ignoring a large number of shelfouts and undermining the objective of increasing sales. Although the dilemma of "crying wolf" is a topic of interest in many fields (e.g., emergency management), the tradeoff between aggressively labeling items as positive versus not labeling enough cases as positive is known as the precision-recall tradeoff in the field of machine learning. In the beginning, AMI chose a high cutoff score to improve the chances the MET members would find an item experiencing a shelfout. As the project matured, the AMI group lowered the threshold for labeling a case as positive. Improving classification accuracy in production settings is an ongoing research topic (Agrawal \& Bonde, 2017).

\section{Application Integration (Mid-January through Mid-March)}

To ensure the output of the model reached the hands of the appropriate associates, the AMI group made use of an application called Smart List that was already deployed at the stores and something with which the store associates were already familiar. Each day at 8:00 am associates retrieve a hand-held device running the Smart List application. They check the application for a list of tasks to start the day. The Smart List application stores all relevant information at the individual SKU level, a feature that was critical for the proper deployment of the project. AML used this application to send out targeted tasks for associates to check on a potential shelfout incident. The only modification AMI had to incorporate in the Smart List application was to add a "Yes" and "No" buttons, which the associates used to record the accuracy of the model's predictions.

Adding the Yes/No buttons on the Smart List application, although it does not seem complex, was one of the roadblocks that took extra time during the project. This was due to the complexity of the existing framework of the application. The AMI group members were not familiar with the deployment process of the team responsible for maintaining the Smart List application, as every team has its own standard operating procedures. The AMI group had to learn the deployment procedures of the other team and follow the rigorous quality control and testing procedures. These procedures introduced multiple layers of approvals and checks throughout the deployment process, which further extended the process.

\section{Second Pilot Test (March through Mid-April)}

The next step in the process was to operationalize these predictions across multiple stores. For the first pilot test, the AMI group utilized data from BigQuery and ran the models through Jupyter Notebooks on their laptops. For the second pilot test, the AMI group developed and executed everything using Google's cloud platform. They migrated all the data they needed from across multiple functional teams into Google's cloud platform. The maximum amount of data size they used for their inference pipeline and training consisted of around 66 million SKUs, which is the number of SKUs per store multiplied by the number of stores the team was working with and resulted in a table size of approximately 80TB just for the sales. Adding in the supply chain data increased the size substantially because the system adds a row in the related supply chain management database every time an item is handled on its way to the store shelf. With over seven years of historical transactional data, the source tables were very massive. The AMI group used a more manageable data size of about 50GB to train the model. The team built more about 13 SQL queries inside Google's BigQuery as part of the data preparation process, which was a huge technical innovation compared to their earlier processes. Data were loaded 
into Google's Cloud Computing Platform cloud from other systems and were extracted from Google Cloud Storage system, which was then fed into the model; then a service picked up those inferences out of BigQuery and pushed them into the "Smart List" devices at each store.

\section{Phased Rollout (April through July)}

After the short second pilot project, the AMI group decided to implement the project in phases. They started with one store and quickly expanded it to five stores. Going from one store to five, they encountered small impediments. One example was with the Smart List application's screen layout. Debriefing with the associates led to the discovery that they were not making full utilization of the extra screen layout of the app. This led to multiple iterations of screen layouts with active feedback from associates to ensure full utilization of the application. With the modifications incorporated into the Smart List application and a smooth utilization of the application producing the desired outcome, the project was implemented at 50 stores with plans to expand it to all stores within a year.

\section{LESSONS LEARNED}

With the project implemented in a limited number of stores, the team reflected on what they learned from this project.

\section{Hybrid Cloud}

One of the reasons this project succeeded is that prior to this project starting, Home Depot adopted a hybrid cloud architecture. In a hybrid cloud environment, some systems that once resided on premises reside in the cloud while other systems remain on premises. Home Depot migrated its Enterprise Data Warehouse (EDW) to Google's BigQuery, one of the data sources for the shelfout application, but other systems like those required to run the stores remained on premises. It supports reporting and analysis efforts and is separate from the systems and databases required to conduct daily business operations.

\section{Service-Level Agreements}

The reliance of data coming from many sources introduced the challenge that the data might not arrive in time to produce the daily shelfout predictions. Service-level Agreements (SLAs) helped manage expectations between the groups AMI needed to get data from and the groups that AMI supplied data to in the form of predictions. An SLA is a written agreement between an entity providing a service (e.g., supply chain group, point-of-sales data group) and a service user (e.g., the AMI group) documenting expectations for quality, availability, and responsibilities. SLAs are often part of contracts but can be useful for managing projects like this one. In addition to SLAs between the various data management teams and the AMI group, there was an SLA between the AMI group and the Home Depot stores. One part of the SLA between the AMI group and the stores was that shelfout predictions would arrive no later than 8:00 am. To account for the interdependencies associated with pulling data from multiple systems, the data pipeline process began each morning at 6:00 am, two hours before the deadline, with SQL preprocessing steps pulling data from the production systems (sales, inventory, etc.) and the EDW.

The process uploaded the results of these steps into a Google Cloud Platform (GCP) storage bucket, which functions like a folder on a personal computer. GCP is the umbrella term for the cloud-based computing services Google offers to developers, including Google BigQuery, Google Image Recognition services, Google Compute Engine, and so forth. Only after the necessary data elements were in the storage bucket could the inference process begin. When a required data element did not arrive in the bucket on time, the AMI group's system sent an email to the responsible party, identified in the SLA, requesting immediate attention to the issue. Predictions not ready before 8:00 am were not sent to the stores. The team established the 8:00 am deadline to minimize the cost 
associated with a false positive. Indicating an item is likely experiencing a shelfout when it is not, a false positive, wastes labor and reduces confidence in the predictions. If the system generated too many false positives, associates might ignore predictions, or worse, associates might clear an item in the Smart List application without checking it. SLAs can help integrate information systems within existing companies and when companies merge (De la Harpe \& Thurner, 2019).

\section{Cloud-Application Management Software}

The AMI group used the Kubernetes software system to manage the computing resources required to train the models each week and produce the inferences each day. Kubernetes software supports automating the deployment, scaling, and management of containerized applications (Red Hat, 2020). A containerized application has its code and any code it relies on compiled to be independent of the development environment, which eases portability and scalability. Docker is a popular container creation and management software system (Vaughan-Nichols, 2018). Containerized applications can run outside of a cloud environment on laptops or desktops but are often associated with cloud computing environments. Kubernetes and Docker are open-source technologies all the major cloud vendors support. Kubernetes orchestrates the deployment of containerized applications and the required computing resources. If a process requires 20 copies of a containerized application to run for five minutes and then shut down, Kubernetes can manage that process and restart any containerized applications that might fail. This architecture scaled from supporting the implementation at one store, then five stores, then 50 stores, and then nationally.

Without the combination of Kubernetes, containerized application, and cloud computing, the architecture required to complete the project would have been exponentially more complex and expensive. Model training that would have taken several days on a desktop only took a few hours in the cloud. The inference process that would have taken hours running on local computers lasted minutes in the cloud. Having access to relevant data, robust software in the form of Kubernetes with containerized applications, and ample computing power made the project practical.

\section{Well-Rounded Teams}

The AMI group being in a large organization like Home Depot benefited by having a well-rounded structure. They had their own user interface and user experience personnel, software engineers, data scientists, and people with the other requisite skills needed to see the project through from start to finish. Such access to a qualified workforce within the team significantly reduced the dependency on other teams, which could have extended the project timeline further. Also, the implementation of multiple pilot phases helped the group develop relationships and obtain buy-in from the business side of Home Depot, which runs the store operations. Such buy-in from key stakeholders who understood the value of solving for shelfout in the early phase of the project was vital to the success of this project.

\section{Cross-Functional Collaboration}

Although using an existing application, Smart List, significantly reduced the barriers to change and the time required to train associates, it did introduce an unexpected complication. The AMI group wanted to modify the Smart List application to include a button associates could use to indicate if an item predicted to be experiencing a shelfout was actually experiencing a shelfout. The group responsible for the Smart List application had defined standards for developing, implementing, and testing any changes to the application. The AMI group did not anticipate the amount of time and effort required to meet these standards. Although those "Yes" and "No" buttons look small, they represent a prolonged collaboration between the two groups.

Beyond working with the group responsible for the Smart List application, the AMI group worked with many other groups. This project involved collaborating with groups to extract data from many systems across the company. In addition to working with other IT teams, the project required support from other teams, including the MET that stocks the shelves during the day, the FT that 
does the overnight packing, store operations, space planning, supply chain, and finance. All these teams worked with the AMI group to help make the project a success. This project illustrates the importance of interdisciplinary teamwork as discussed in information systems project management literature (Brooks, 2017).

\section{FUTURE STATE}

With the project rollout going smoothly, the AMI group is considering expanding the project in three broad areas. These are model improvement, enhanced data, and machine learning/artificial intelligence algorithms. This section discusses each of these areas.

\section{Model Improvement}

For the future, the AMI group would like to focus not only on the precision and optimization of the predictions but also on more ways to expand the technique to include features such as sales margin and substitution products, among others. For example, if a customer goes to a Home Depot store to buy a hammer, and all red-colored hammers are experiencing a shelfout but blue hammers are not, the customer will likely buy a blue hammer because a blue hammer is a substitutional product to a red hammer. Shelfouts on highly substitutable products are not that critical to solve. The team would want to incorporate such exception handling knowledge in their models, leading to models that are active learning. Active learning is a machine learning approach that automates the process of selecting data, building models, implementing models, and monitoring model performance. As the team deploys the application in more stores and the available training data grows, they plan to train and deploy the model on an hourly basis instead of a daily basis.

\section{Data}

The AMI group will be working towards finding more advanced sources of data to augment what they currently have in place. One such source is images from cameras and sensor fusion. For a retailer such as Home Depot that has about 2,200 stores, such data extraction could be an expensive endeavor, which is why the AMI group started with using data the MET collected during the early-stage proofof-concept project. External data such as weather forecasts, census data, and general economic data might also prove useful.

\section{Machine Learning/Artificial Intelligence}

During the development of this project, the AMI group built a lot of tooling infrastructure in a machine learning/artificial intelligence platform, which enabled the team to engage in business experiments by creating multiple pilot tests. This platform allowed the team to use the same infrastructure and quickly scale it up as needed for future projects. Furthermore, being a new team, incorporating machine learning for the first time presented them with a huge learning opportunity. They were able to grow through their challenges and create machine learning opportunities for other business units within the company through their successful project implementation. Now AMI is considering how to use the new infrastructure and capabilities. They could continue developing the infrastructure for others to use by building tools that enable other business units to develop a project. They could also focus on educating other business units and becoming advisors in a machine learning/artificial intelligence center of excellence. Machine learning/artificial intelligence has transformed many industries, and there are no indications the rate of change will slow soon (Ferràs et al., 2020). 


\section{CONCLUSION}

This case describes the development and implementation of advanced data analytics software to increase sales at Home Depot by increasing the availability of products on store shelves. The system the company developed relies on cloud computing capabilities and machine learning algorithms. The case illustrates challenges associated with managing the development of big data applications in an enterprise setting by describing challenges associated with implementing software that requires ingestion and processing copious amounts of data in a production setting. 


\section{REFERENCES}

Agrawal, D., \& Bonde, P. (2017). Improving classification accuracy on imbalanced data by ensembling technique. Journal of Cases on Information Technology, 19(1), 42-49. doi:10.4018/jcit.2017010104

Brooks, S. (2017). Interdisciplinary app development project: A case study across three departments. Journal of Cases on Information Technology, 19(3), 15-23. doi:10.4018/JCIT.2017070102

Brunello, A., Gallo, P., Marzano, E., Montanari, A., \& Vitacolonna, N. (2019). An event-based data warehouse to support decisions in multi-channel, multi-service contact centers. Journal of Cases on Information Technology, 21(1), 33-51. doi:10.4018/JCIT.2019010103

Charrington, S. (2018). How ML keeps shelves stocked at Home Depot with Pat Woowong. https://twimlai.com/ twiml-talk-175-how-ml-keeps-shelves-stocked-at-home-depot-with-pat-woowong/

Chuang, H. (2018). Fixing shelf out-of-stock with signals in point-of-sale data. European Journal of Operational Research, 270(3), 862-872. doi:10.1016/j.ejor.2017.10.059

De la Harpe, A., \& Thurner, T. W. (2019). The critical role of information system integration in a horizontal merger. Journal of Cases on Information Technology, 21(4), 1-13. doi:10.4018/JCIT.2019100101

Ferràs, X., Hitchen, E. L., Tarrats-Pons, E., \& Arimany-Serrat, N. (2020). Smart tourism empowered by artificial intelligence: The case of Lanzarote. Journal of Cases on Information Technology, 22(1), 1-13. doi:10.4018/ JCIT.2020010101

Google. (2018). The value proposition for using ML in brick-and-mortar retail stores: Home Depot (Cloud Next '18). https://youtu.be/rF8jtdX-hGo

Home Depot. (2019). The home is where our story begins. https://corporate.homedepot.com/about/history

Red Hat. (2020). What is Kubernetes? https://www.redhat.com/en/topics/containers/what-is-kubernetes

Vaughan-Nichols, S. (2018). What is Docker and why is it so darn popular? https://www.zdnet.com/article/ what-is-docker-and-why-is-it-so-darn-popular/

Herbert Remidez is an Associate Professor at the University of Dallas. He had taught courses in predictive modeling, data management, quantitative methods, advance business analytics, IT project management and IT strategy and governance. Herbert's research interests include machine learning, predictive analytics, and aggregating information to improve project duration and cost estimates. He has published journal articles in Communications of the ACM, International Journal of Knowledge and Learning, Computers in the Schools, and the International Journal of e-Collaboration.

Sri Beldona is a Professor of Management. Prior to his appointment at the University of Dallas, he worked as Vice President of Strategic Planning at Marketing Management, Inc. in Fort Worth. He received his Doctorate in Business Administration from Temple University, where he concentrated on International Business and Strategic Management. His areas of expertise include product and brand management, consumer behavior, international marketing, and business analytics. 